\title{
Patellar tendon rupture
}

\begin{abstract}
Patellar tendon rupture is a rare pathology with few cases series reported in literature. The aim of this study is to describe the epidemiological, clinical profile of patellar tendon ruptures as well as its operative management and outcome. We carried out a retrospective descriptive study involving 11 cases of patellar tendon rupture managed between 2012 and 2018 at the department of Traumatology and Orthopedic surgery A of Hassan II Teaching Hospital, Fez. It is an infrequent injury potentially occurring at any age in life with a clear predominance in young male adults.
\end{abstract}

All cases pertain to an acute post-traumatic rupture. Diagnosis was made preoperatively on physical examination in most patients. Surgery found a full body mid substance ruptures in most cases with a distal tear only in the $33.3 \%$ of cases.

Surgery is the mainstay treatment. It involves repair using vicryl 2 sutures reinforced by a wire frame in most cases patients: transosseous sutures augmented with a steel wire frame or an autologous semitendinosus graft.

Functional outcomes were evaluated using Swik's criteria, with 58.3\% cases exhibiting excellent or good results.

Thus the authors conclude that acute patellar tendon ruptures affect mainly young active males with surgery being the mainstay treatment that guarantees favorable outcome depending on the time of repair and postoperative patella height.
Volume 10 Issue 5 - 2018

\author{
Boubacar Soumare,' Marzouki Amine,' \\ Sériba Sidibe,' Bah Souleymane,' Abdoul \\ Salam Diarra, ${ }^{2}$ Fawzi Boutayeb' \\ 'Service de Traumatologie etd'Orthopédie Adu CHU Hassan II/ \\ Faculté de Médecine et de harmacie/Université \\ 'Laboratoire d'Epidémiologie et de santé Publique/Faculté de \\ Médecine et de Pharmacie de/Université Sidi Mohammed Ben \\ Abdellah de Fès/Maroc
}

\section{Correspondence: Boubacar Soumare, Médecin Résident traumatologie-orthopédie A/Faculté de Médecine et de Pharmacie de /Université Sidi Mohammed Ben Abdellah de Fès/ Maroc, Tel 002126288I5726, Email docsowm19@gmail.com}

Received: August 10,2018 | Published: October 26, 2018

Keywords: orthopedic surgery, hospital, patients, epidemiological, traumatology

\section{Introduction}

Patellar tendon can rupture following sudden forceful movement Dynamic forces applied during sports are often higher than the static resistance of the tendon. In a healthy male (without patellar tendinopathy or underlying systemic disease), the patellar tendon ruptures when a force 17.5 times the body weight is applied. To illustrate, climbing a flight of stairs loads the patellar tendon by a force 3.3 times the body weight ${ }^{1}$ whereas jumping loads the tendon up 7 to 8 times the body weight. Acute patellar tendon ruptures are infrequent yet represent a severe potentially debilitating injury of the adult male with nearly $80 \%$ of cases involving patients under 40 years old. ${ }^{2}$

\section{Patients and methods}

We retrospectively reviewed the folders of 11 patients managed at the Department of Traumatology and Orthopedics Surgery A, Hassan II Teaching Hospital, Fez between May 2012 and May 2018. Only patients with an acute patellar tendon rupture were included in this study thus all patients with spontaneous rupture were excluded. All patients underwent emergent repair. Clinical presentation was dominated by patellar gapping, in addition to intense pain and inability to actively extend the knee. Plain x-ray, AP and lateral views, obtained in all cases to rule out any fractures or bony avulsions allows to measure patella height using the Caton-Deschamps index. Ultrasound was performed in 7 patients whereas 4 patients underwent MRI as part of preoperative workup.

All patients underwent repair under spinal or general anesthesia on an ordinary table in supine position with an upper thigh tourniquet to aid exposure. We performed an anterior approach with a midline skin incision extending from the patella apex to the tibial tubercle (TT). After careful dissection of tendinous sheath over the unruptured portion, the tendon was released along its length and all its edges. The ends of the tear were careful debrided as the tendon was brought together and sutured end to end and tied using the Judet suture technique. ${ }^{3}$ To protect against failed repair, a patellar tendon frame was made with either a steel wire passing through the patella and the TT, or an autologous tendon frame using semitendinosus in one case. Post-operative immobilization using removable splint was worn for a duration of 45days in all patients with brace-ambulation and weight bearing advocated on the 21 st post-operative day. Rehabilitation began on the 3 rd postoperative week with passive range of flexion up to $70^{\circ}$ for up to 45 days. Patients were regularly reviewed with routine physical and $\mathrm{x}$-ray. After a minimum follow-up of 6 months we measured functional outcome using the Siwek criteria ${ }^{4}$ based on two elements: range of motion and quadriceps tone. Included in this series was a case of bilateral rupture in a young amateur sportsman of 25 years who sustained the injury after being stabbed.

\section{Results}

$83.3 \%$ of cases involved patients aged less than 40years old, $41.7 \%$ were amateur sportsmen, $25 \%$ of whom were practicing daily and $91.7 \%$ were male. Tobacco and alcohol were the most common toxic habits with $25 \%$ and corticosteroids use was observed in $41.7 \%$ of patients.

Rupture was traumatic in all cases with direct impact (91.7\%): $33.3 \%$ cases following fall, $25 \%$ of cases sports related injury, $8.3 \%$ due to accidents and $33.3 \%$ of cases involving stabbing injuries.

Pain and knee hemarthrosis was found in all patients with an open wound in 3 cases. Functional impairment was total in only $58.3 \%$ of cases. Pre-operative Caton-Deschamps showed patella alta in $83.3 \%$ of the patients which was corrected postoperatively. 
Majority of the patients underwent emergent repair under spinal anesthesia in $75 \%$ of cases whereas $25 \%$ were operated under general anesthesia. The average admission time was 3hours (between 1-6hours). The mean hospital stay was 2days (ranging from 1-4days).

A midline anterior approach was used in all cases with peroperative discovery of: complete tear near the tip of the patella $(33,3 \%)$, midsubstance tear tendon $(33,3 \%)$, and near the TT in $25 \%$ of cases.

Seventy-five percent of patients underwent end to end repair using the double KESLER suture technique protected by a steel wire frame in $33.3 \%$ of patients or autologous semitendinosus tendon frame in $33.3 \%$ whereas $25 \%$ of patients benefited repair using transosseous patella drills using vicryl 2 sutures in 2 cases and wire frame augmentation in 1 case.

Associated injuries included 1 case of patella fracture, 3 cases of meniscal injury and 1 case of anterior cruciate ligament (ACL) injury. After a minimum follow-up of 6months we noted flexion deficit of 10 to $20^{\circ}$ in 3 patients whereas 2 patients had an extension lag of 5 to $10^{\circ}$ compared to the uninjured side. According to Swik's criteria, these represented excellent or good results in $58 \%$ of cases(Figure 1).
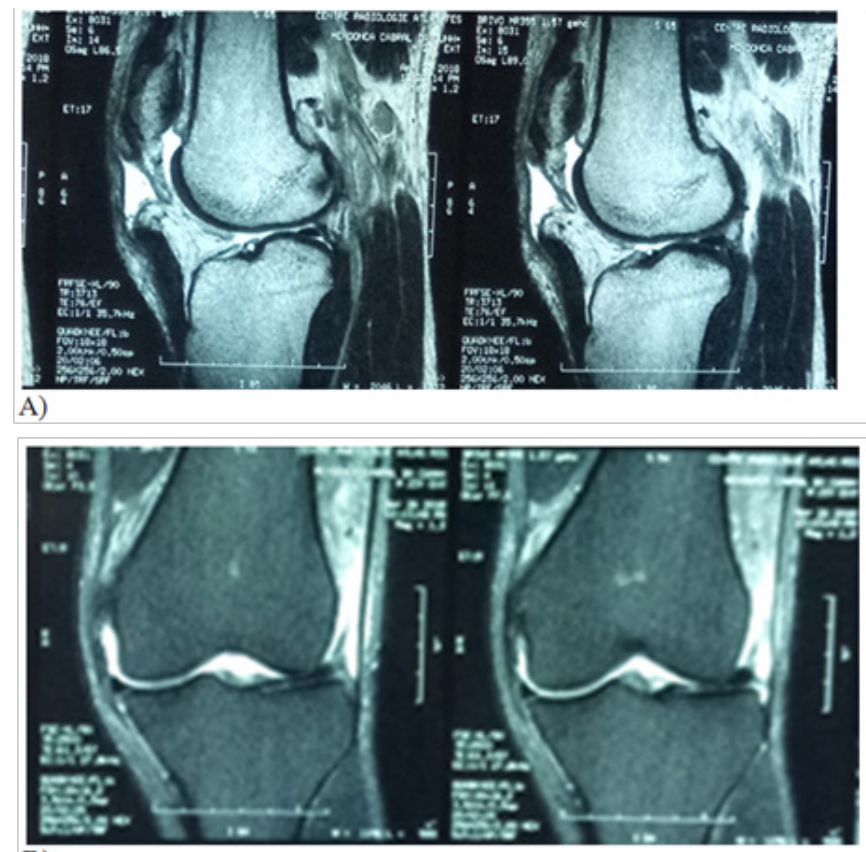

B)

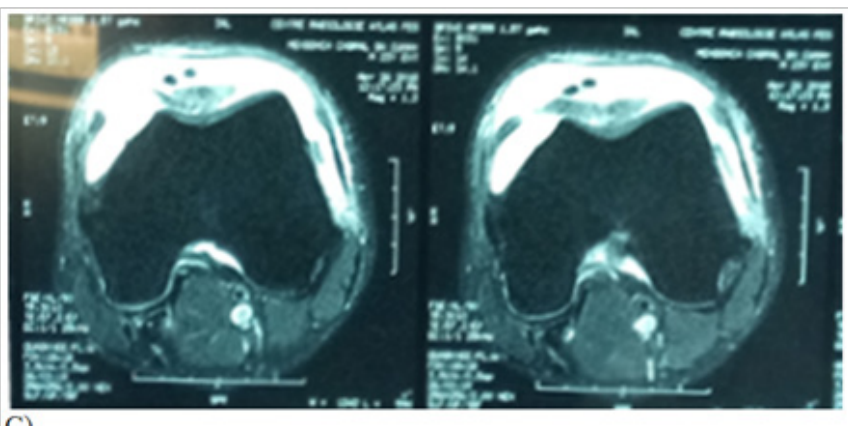

C)
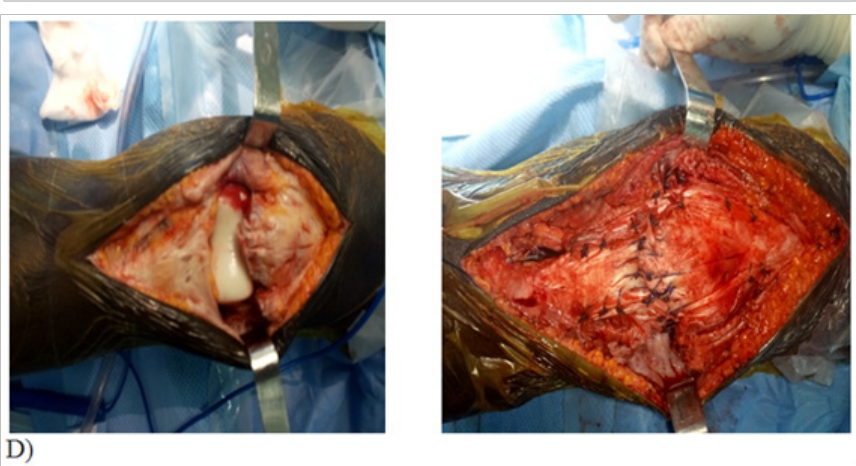

Figure I Case: Patient 28 years old male, 10 day history of sports injury with complete patella tear accident: we proceeded to a repair using trans-osseous patella suturing with vicryl 2 and reinforcement semitendinosus.

A-T2 sagittal section

B, C etc- coronal and axial section in particular sequence of proton density

D- per-operative image. 


\section{Discussion}

The actual incidence of patellar tendon rupture is not known. It accounts for the $3^{\text {rd }}$ most frequent cause of adult native extensor mechanism ruptures after patella fractures and quadriceps tendon ruptures. ${ }^{5}$ It is often seen in young males under 40years old who rupture their extensor mechanism during sports activities. ${ }^{6}$ This is corroborated by our results with $83.3 \%$ of patients under 40 and a male predominance of $91.7 \%$.

Rupture of the patellar tendon is often as a result of a direct trauma during the sports activity as was the case in our series with predominant causes being falls $33.3 \%$, sports injury in $25 \%$ and assaults in $33.3 \%$. Underlying tendinopathy or systemic disease and even repetitive micro trauma called "jumper's knee" are also believed to be major risk factors of this pathology ${ }^{7}$ as the prevalence of "jumper's knee" is more frequent in contact sports requiring high level of speed and strength of leg extensor mechanisms such as volleyball, football and athletics. ${ }^{8}$ In our series we observed a high prevalence of jumpers 'knee in $41.7 \%$ of our amateur athlete population whereas substance abuse like tobacco and alcohol was noted in $25 \%$ and long corticosteroid use in $41.7 \%$ of the patients.

Clinically, patients present with acute knee pain, edema, and functional impairment and especially a palpable patellar gap and a failure to maintain active knee extension on physical examination. ${ }^{9}$ Plain X-rays could reveal "patella alta" 10 The use of complementary diagnostic imaging such as ultrasound and MRI is necessary if there is surrounding diagnosis and allows associated injury assessment ${ }^{11,12}$ as evidenced by our study.

Current literature is unanimous on the emergent surgical repair of patella tendon ruptures. When the diagnosis of patellar tendon rupture is made, surgical repair should be considered regardless of the time of the rupture. ${ }^{9}$

Several techniques have been previously described for the repair which need to be used on a case-by-case basis. ${ }^{13}$ Surgical exploration during repair found rupture: close to the patella border in $33.3 \%$, midsubstance tear $33.3 \%$, and a complete tear near the TT in $25 \%$ of cases. Our results are similar to those of Ait Selmi, ${ }^{14}$ rupture usually occurs close to the patella border in $43 \%$ of cases, and the subpatellar region in $14 \%$ of cases.

Several techniques have been previously described for repair which need to be used on a case-by-case basis ${ }^{13}$ and in general, simple sutures are not biomechanically sound to allow for early motion. They need to be protected through one or several augmentation techniques using non-resorbable sutures instead of a metallic frame ${ }^{9}$ although in our series we found $33.3 \%$ of cases involved a steel wire frame. This augmentation technique is not without drawbacks. First the rigidity of construct, its tendency to fail and need for secondary surgery for hardware removal. Also its tendency to tilt the patella in the sagittal plane, or even to lower it. ${ }^{9}$ We also carried out an autologous tendon reinforcement using semitendinosus in $33.3 \%$ of cases as reported by many authors although we found large variety of autologous reinforcement techniques in literature (semi-tendinous, gracile semitendinosus (GST) graft, fascia lata...). As in our series, most authors advocate postoperative immobilization, ${ }^{15}$ although a select few have reported excellent result with early motion. ${ }^{16,17}$ This they argue contributes to quadriceps muscle atrophy and joint stiffness. For our part, rehabilitation was started on the 45th postoperative day.

Functional outcomes are measured using several knee scoring systems available in literature an indirect indication of lack of reference. Our results are similar to those reported in literature .Despite numerous technically challenges, we reported favorable outcomes with 58, 3\% excellent and good results according to Swik's criteria. Outcome depends largely on the age of the patients, early repair and finally the technique of repair which confers adequate strength of construct to restore early motion and patellar height. The diversity of techniques of repair does not allow for clear cut guidelines regarding operative management of patella tendon ruptures.to a uniform therapeutic choice. Nonetheless, regardless of the technique of repair used, satisfactory outcome is usually attributed to early repair.

\section{Conclusion}

Patellar tendon rupture is an uncommon injury in the broad spectrum of extensor mechanism ruptures affecting young active males. Diagnosis is most often clinical. Management is surgical. (Early repair guarantees good functional outcome.

\section{Acknowledgements}

None.

\section{Conflict of interest}

The author declares no conflict of interest.

\section{References}

1. Nordin M, Frankel VH. Biomechanics of the knee. In: Nordin M, Frankel $\mathrm{VH}$, editors. Basic biomechanics of the musculoskeletal system. 2nd ed. Philadelphia: Lea \&Febiger; 1989:115-34.

2. Siwek CW, Rao JP. Ruptures of the extensor mechanism of the knee joint. J Bone Joint Surg. 1981;63:932-937.

3. Caldas MTL, Gustavo HSB, Manuela BFB. Simultaneous bilateral rupture of the patellar ligament in chronicrenal patient, case report. Rev bras ortop. 2013;48(5):455-459.

4. Siwek CW, Rao JP. Ruptures of the extensor mechanism of the knee joint. J Bone Joint Surg Am. 1981;63(6):932-937.

5. Annunziata $\mathrm{C}$, Ignacio E, Patellar. Tendon Rupture. E-Medicine. 2006:1-10.

6. Kallersmann R, Blattert TR, Weckbach A. Bilateral patellar tendon rupture without predisposing systemic disease or steroid use: a case report and review of the literature. Arch Orthop Trauma Surg. 2005; 125(2):127-133.

7. Kannus P, Natri A. Etiology and pathophysiology of tendon ruptures in sports. Scand J Med Sci Sports. 1997;7(2):107-112.

8. Lian B, Engebresten L, Bahr R. Prevalence of jumper's knee among elite athletes from different sports: a cross-sectional study. Am J Sports Med. 2005;33(4):561-567.

9. Boggione $\mathrm{C}$, Marmorat JL. Traitement des ruptures totales du tendon rotulien. J Traumatol. Sport. 2004;21(4):204-217.

10. Rose PS, Frank J, Frassica. Atraumaticbilateralpatellar tendon rupture. $J$ Bone Joint Surg Am. 2001;83(A9):1382-1386.

11. Bianchi S, Cohen M, Jacob D. Les tendons : lésions traumatiques. Journal de Radiologie. 2005;86(12):1845-1857.

12. Yu Joseph S, PetersilgeCh, Sartoris D. MR Imaging of injuries of extensormechanism of the knee. Radiographics. 1994;14:541-551.

13. Bumbasirevic M, Lesic A. Acute injuries of the extensormechanism of the knee. Current Orthopaedics. 2005;19(1):49-58. 
14. Ait Si Selmi T, Neyret P, Rongleras F. Ruptures de l'appareil extenseur du genou et factures de la rotule. EMC Techniques chirurgicalesOrthopédieTraumatologie. 1990;44-730.

15. Siwek CW, Rao JP. Ruptures of the extensor mechanism of the knee joint. J Bone JtSurg. 1981;63(6):932-937.
16. Bhargava SP, Hynes MC, Dowell JK.Traumatic patella tendon rupture: early mobilisation following surgical repair. Injury. 2004;35(1):76-79.

17. Enad JG, Larry LL. Patellar tendon repair: postoperative treatment. Arch Phys Med Rehabil. 2000;81(6):786-788. 